International Journal of Pure and Applied Mathematics

Volume 94 No. 4 2014, 515-524

ISSN: 1311-8080 (printed version); ISSN: 1314-3395 (on-line version)

url: http://www.ijpam.eu

doi: http://dx.doi.org/10.12732/ijpam.v94i4.6

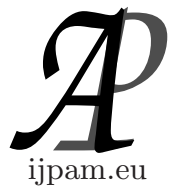

\title{
ON THE MOMENTS OF STOCHASTIC ANNUITIES
}

\author{
A.R. Mugdadi ${ }^{1}$, Ibrahim A. Ahmad ${ }^{2}$ \\ ${ }^{1}$ Department of Mathematics and Statistics \\ Jordan University of Science and Technology \\ Irbid, JORDAN \\ ${ }^{2}$ Department of Statistics \\ Oklahoma State University. \\ Stillwater, OK 74078, USA
}

\begin{abstract}
The interest (discount) rate of annuity is a random variable, which is called stochastic. For stochastic annuities, also known as annuities under random rates of interest, we derive some moments, moments inequalities, and moments limits. When the discount rates are bounded, a strong convergence result is given showing that a stochastic annuity converges to its mean value as the number of years tend to infinity. Hence, stochastic annuities satisfy a type of strong law of large numbers.
\end{abstract}

AMS Subject Classification: 62P05, 60H30

Key Words: stochastic annuity, moments inequalities, strong limit theorem, actuarial science, probability

\section{Introduction}

An annuity is a series of payments made at equal intervals of time for a finite (or an infinite) number of periods. When the interest rates are affect its growth are random variables, the annuity is "stochastic", or is "under random rate of interest." Let $Y_{1}, Y_{2}, \ldots, Y_{n}$ be $n$ variables such that $Y_{i} \geq 0, i=1,2, \ldots, n$

Received: February 20, 2014

(C) 2014 Academic Publications, Ltd.

$\S$ Correspondence author url: www.acadpubl.eu 
representing the random effective rates of interest over $n$ random periods which is the life of the annuity. to find how the accumulated value of this annuity can be measured, one must decide on how the interest rates will operate; if $Y_{i}$ is used for period $i$ regardless of when this payment is made, then the accumulated value of one unit payment is given by (cf. Bowers, et. al [1]):

$$
A_{n}=\sum_{i=1}^{n} \prod_{l=1}^{i}\left(1+Y_{n-l+1}\right) .
$$

While if $X_{i}$ is used for payment made at time $i$ over all $i$ periods, the we have

$$
A_{\bar{n}}^{*}=\sum_{i=1}^{n}\left(1+Y_{n-i+1}\right)^{i} .
$$

Another function, the present value of the annuity is often of interest and is defined by

$$
P_{\bar{n}}=\sum_{i=1}^{n} \prod_{l=1}^{i}\left(1+Y_{l}\right)^{-1}
$$

in the first situation, while the second is given by

$$
P_{\bar{n}}^{*}=\sum_{i=1}^{n}\left(1+Y_{i}\right)^{-i}
$$

Another representation for the present value is possible via the "random discount rate" $X_{i}$ related to the random interest rate $y_{i}$ by the relation $1-X_{i}=$ $\left(1+Y_{i}\right)^{-1}$ or $Y_{i}=\frac{X_{i}}{1-X_{i}}$ and $0 \leq X_{i} \leq 1$. Thus, we get

$$
P_{\bar{n}}=\sum_{i=1}^{n} \prod_{l=1}^{i}\left(1-X_{l}\right)
$$

and

$$
P_{\bar{n}}^{*}=\sum_{i=1}^{n}\left(1-X_{i}\right)^{i} .
$$

In this note, we concentrate on (1.5) and indicate corresponding results for (1.6). We shall assume throughout that $X_{1}, X_{2}, \ldots, X_{n}$ are independent random variables such that $\alpha_{i} \leq X_{i} \leq \beta_{i}$, for constants $0 \leq \alpha_{i} \leq \beta_{i}, i=1,2, \ldots, n$. stochastic annuities is a field in actuarial science that is seeing an increasing attention recently. Several authors attempted calculating the mean and the 
variance of the present (or accumulated) value and found that there is appreciable difficulty in so doing, cf. Kling, Ruez, and Rub [6], Dufresne [4], Burnecki, Marciniuk and Weron [3], Panjer and Belhouse [1] and [2], Promislow [9] and Boyle [2] for some work related to this issue.

In the current investigation we offer bounds of the mean and variance of $P_{\bar{n}}$. Also, when the discount rates have same mean we obtain limit expressions of the variance as well. Finally we give sufficient conditions such that $P_{\bar{n}}-E P_{\bar{n}} \rightarrow 0$ with probability one, as $n \rightarrow \infty$

section Moments Inequalities

In this section, we present relations of moments and moments inequalities for $P_{n}$ (and $P_{n}^{*}$ given in (1.3) and (1.4) or equivalently by (1.5) and (1.6) respectively . We shall assume that the $X_{i}$ 's are independent.

Theorem 1. (i) If $E\left(X_{i}\right)=\mu$ for all $i=1,2, \ldots, n$, then

$$
E\left(P_{\bar{n}}\right)=\frac{1-\mu}{\mu}\left[1-(1-\mu)^{n}\right]=E_{n}(1-\mu), \text { say. }
$$

(ii) In general, if $\bar{\mu}_{n}=\max _{1 \leq i \leq n} \mu_{i}$ and $\bar{\mu}_{n}=\min _{1 \leq i \leq n} \mu_{i}$, and $\mu_{i}=E\left(X_{i}\right)$, $i=1,2, \ldots, n$, then

$$
E_{n}\left(1-\bar{\mu}_{n}\right) \leq E\left(P_{\bar{n}}\right) \leq E_{n}\left(1-\underline{\mu}_{n}\right)
$$

Proof. (i) Follows directly from the fact that

$$
E\left(P_{\bar{n}}\right)=\sum_{i=1}^{n}(1-\mu)^{i}=\frac{1-\mu}{\mu}\left[1-(1-\mu)^{n}\right]
$$

(ii) Follows directly from the fact that

$$
\sum_{i=1}^{n}\left(1-\bar{\mu}_{n}\right)^{i} \leq E\left(P_{\bar{n}}\right)=\sum_{i=1}^{n} \prod_{l=1}^{i}(1-\mu) \leq \sum_{i=1}^{n}\left(1-\underline{\mu}_{n}\right)^{i}
$$

Note that if $E\left(X_{i}\right)=\mu, \mu \in(0,1)$, then $\lim _{n \rightarrow \infty}=E\left(P_{\bar{m}}\right)=\frac{1-\mu}{\mu}$, a result known for nonstochastic annuities, cf. Kellison [5], p69. Also, if $\underline{\mu}_{n} \rightarrow \underline{\mu}$ and $\bar{\mu}_{n} \rightarrow \bar{\mu}$, where $\mu$ and $\bar{\mu}$ are finite, then, in general,

$$
b=\frac{1-\bar{\mu}}{\bar{\mu}} \leq \lim _{n \rightarrow \infty} E\left(P_{\bar{n}}\right) \leq \frac{1-\underline{\mu}}{\underline{\mu}}=a
$$


Theorem 2. (i) If for $i=1,2, \ldots, n, E\left(X_{i}\right)=\alpha$ and $E\left(X_{i}^{2}\right)=\alpha_{(2)}$, then as $n \rightarrow \infty, E P_{n} \rightarrow \frac{1-\alpha}{\alpha}$ and

$$
\operatorname{Var}\left(P_{\bar{n}}^{2}\right) \rightarrow \frac{\alpha_{(2)}-\alpha^{2}}{\alpha^{2}\left(2 \alpha-\alpha_{(2)}\right.},
$$

as $n \rightarrow \infty$, provided $\alpha \in(0,1)$.

(ii) In general, if $\bar{\mu}_{n}^{(2)}=\max _{1 \leq i \leq n} \mu_{i}^{(2)}$ and $\underline{\mu}_{n}^{(2)}=\min _{1 \leq i \leq n} \mu_{i}^{(2)}$, then

$$
B_{n} \leq E\left(P_{\bar{n}}\right)^{2} \leq A_{n},
$$

where,

$$
\begin{aligned}
& A_{n}=\frac{\left(1-2 \underline{\mu}_{n}+\bar{\mu}_{n}^{(2)}\right)}{2 \underline{\mu}_{n}-\bar{\mu}_{n}^{(2)}}\left[1-\left(1-2 \underline{\mu}_{n}+\bar{\mu}_{n}^{(2)}\right)^{n}\right] \\
& +\frac{2\left(1-\underline{\mu}_{n}\right)}{\underline{\mu}_{n}}\left(1-2 \underline{\mu}_{n}+\bar{\mu}_{n}^{(2)}\right) \\
& {\left[\frac{1-\left(1-2 \underline{\mu}_{n}-\bar{\mu}_{n}^{(2)}\right)^{n}}{2 \underline{\mu}_{n}-\bar{\mu}_{n}^{(2)}}-\frac{\left(1-\underline{\mu}_{n}\right)^{n}-\left(1-2 \underline{\mu}_{n}+\bar{\mu}_{n}^{(2)}\right)^{n}}{\left(1-\underline{\mu}_{n}\right)\left(\underline{\mu}_{n}-\bar{\mu}_{n}^{(2)}\right)}\right],}
\end{aligned}
$$

and $B_{n}$ is obtained from $A_{n}$ after replacing $\underline{\mu}_{n}$ by $\bar{\mu}_{n}$ and $\bar{\mu}_{n}^{(2)}$ by $\underline{\mu}_{n}^{(2)}$.

Proof. We start by proving (ii). Let $T_{n, i}=\prod_{j=1}^{i}\left(1-X_{j}\right), i=1,2, \ldots, n$. Thus

$$
\begin{aligned}
E\left(P_{\bar{n}}\right)^{2}= & E\left(\sum_{i=1}^{n} T_{n, i}\right)^{2} \\
= & \sum_{i=1}^{n} E T_{n, i}^{2}+2 \sum \sum_{i<i^{*}} E T_{n, i} T_{n, i^{*}} \\
= & \sum_{i=1}^{n} E \prod_{i=1}^{n}\left(1-X_{j}\right)^{2}+2 \sum_{i<i^{*}} E \prod_{j=1}^{i}\left(1-X_{j}\right)^{2} \prod_{l=i+1}^{i^{*}}\left(1-X_{l}\right) \\
= & \sum_{i=1}^{n} \prod_{j=l}^{i}\left(1-2 \mu_{n-j}+\mu_{n-j}^{(2)}\right) \\
& +2 \sum_{i<i^{*}} \prod_{j=1}^{i}\left(1-\mu_{n-j}+\mu_{n-j}^{(2)}\right) \prod_{l=i+1}^{i^{*}}\left(1-\underline{\mu}_{n-l}\right)
\end{aligned}
$$




$$
\begin{aligned}
& \leq \sum_{i=l}^{n}\left(1-2 \underline{\mu}_{n}+\bar{\mu}_{n}^{(2)}\right)^{i}+2 \sum \sum_{i<i^{*}}\left(1-2 \underline{\mu}_{n}+2 \bar{\mu}_{n}^{(2)}\right)^{i}\left(1-\underline{\mu}_{n}\right)^{i^{*}-i} \\
& =I_{1 n}+I_{2 n}, \text { say. }
\end{aligned}
$$

Now,

$$
I_{1 n}=\frac{\left(1-2 \underline{\mu}_{n}+\bar{\mu}_{n}^{(2)}\right)}{2 \underline{\mu}_{n}-\bar{\mu}_{n}^{(2)}}\left[1-\left(1-2 \underline{\mu}_{n}+\bar{\mu}_{n}^{(2)}\right)^{2}\right],
$$

and

$$
\begin{aligned}
& I_{2 n}=2 \sum_{i=1}^{n} \sum_{i^{*}=i+1}^{n}\left(1-2 \underline{\mu}_{n}+\bar{\mu}_{n}^{(2)}\right)^{i}\left(1-\underline{\mu}_{n}\right)^{i^{*}-i} \\
& =2 \sum_{i=1}^{n}\left(1-2 \underline{\mu}_{n}+\bar{\mu}_{n}^{(2)}\right)^{i} \frac{\left(1-\underline{\mu}_{n}\right)\left(1-\left(1-\underline{\mu}_{n}\right)^{n-i^{*}}\right.}{\underline{\mu}_{n}} \\
& =\frac{2\left(1-\mu_{n}\right)}{\mu_{n}} \sum_{i=1}^{n}\left(1-2 \underline{\mu}_{n}+\bar{\mu}_{n}^{(2)}\right)^{i}-\frac{2\left(1-\underline{\mu}_{n}\right)^{n}}{\underline{\mu}_{n}} \sum_{i=l}^{n}\left(\frac{1-2 \underline{\mu}_{n}+\underline{\mu}_{n}^{(2)}}{1-\underline{\mu}_{n}}\right)^{i} \\
& =\frac{\left(1-\underline{\mu}_{n}\right)\left(1-2 \underline{\mu}_{n}+\bar{\mu}_{n}^{(2)}\right)}{\underline{\mu}_{n}\left(2 \underline{\mu}_{n}-\bar{\mu}_{n}^{(2)}\right)}\left[1-\left(1-2 \underline{\mu}_{n}+\bar{\mu}_{n}^{(2)}\right)^{n}\right]-\left(1-\underline{\mu}_{n}\right)^{n} \text {. } \\
& \frac{1-2 \underline{\mu}_{n}+\bar{\mu}_{n}^{(2)}\left(1-\underline{\mu}_{n}\right)}{1-\underline{\mu}_{n}}\left[\frac{\left(1-\underline{\mu}_{n}\right)^{n}-\left(1-2 \underline{\mu}_{n}+\bar{\mu}_{n}^{(2)}\right)^{n}}{\left(1-\underline{\mu}_{n}\right)^{2}\left[\underline{\mu}_{n}-\bar{\mu}_{n}^{(2)}\right]}\right] \\
& =\frac{2\left(1-\underline{\mu}_{n}\right)}{\underline{\mu}_{n}}\left(1-2 \underline{\mu}_{n}+\bar{\mu}_{n}^{(2)}\right) \text {. } \\
& {\left[\frac{1-\left(1-2 \underline{\mu}_{n}+\bar{\mu}_{n}^{(2)}\right)^{n}}{2 \underline{\mu}_{n}-\bar{\mu}_{n}^{(2)}}-\frac{\left(1-\underline{\mu}_{n}\right)^{n}-\left(1-2 \underline{\mu}_{n}+\bar{\mu}_{n}^{(2)}\right)^{n}}{\left(1-\underline{\mu}_{n}\right)^{2}\left(\underline{\mu}_{n}-\mu_{n}^{(2)}\right)}\right] .}
\end{aligned}
$$

From (2.8) and (2.9) it follows that $E\left(P_{\bar{n}}\right)^{2} \leq A_{n}$. Next, let $\bar{\mu}_{n}$ and $\underline{\mu}_{n}^{(2)}$ replace $\underline{\mu}_{n}$ and $\bar{\mu}_{n}^{(2)}$, respectively, we obtain exactly as above that

$$
E\left(P_{n}\right)^{2} \geq L_{1 n}+L_{2 n}=B_{n}
$$

where,

$$
L_{1 n}=\frac{1-2 \bar{\mu}_{n}+\underline{\mu}_{n}^{(2)}}{2 \bar{\mu}_{n}-\underline{\mu}_{n}^{(2)}}\left[1-\left(1-2 \bar{\mu}_{n}+\underline{\mu}_{n}^{(2)}\right)^{n}\right]
$$

and 


$$
\begin{aligned}
L_{2 n}=\frac{2\left(1-\bar{\mu}_{n}\right)}{\bar{\mu}_{n}}\left(1-2 \bar{\mu}_{n}+\underline{\mu}_{n}^{(2)}\right)[1 & \left.-\left(1-2 \bar{\mu}_{n}+\underline{\mu}_{n}^{(2)}\right)^{2}\right] \\
& -\frac{\left(1-\bar{\mu}_{n}\right)^{n}-\left(1-2 \bar{\mu}_{n}+\underline{\mu}_{n}^{(2)}\right)^{n}}{\left(1-\bar{\mu}_{n}\right)\left(\bar{\mu}_{n}-\underline{\mu}_{n}^{(2)}\right)} .
\end{aligned}
$$

We now prove part (i)

$$
E\left(P_{\bar{n}}\right)=\sum_{i=1}^{n} \prod_{j=1}^{i}\left(1-\mu_{j}\right)=\sum_{i=1}^{n}(1-\alpha)^{i}=\frac{(1-\alpha)\left[1-(1-\alpha)^{n}\right]}{\alpha}
$$

Thus, we have

$$
\lim _{n \rightarrow \infty}\left[E\left(P_{\bar{n}}\right)\right]^{2}=\left(\frac{1-\alpha}{\alpha}\right)^{2} .
$$

Next, let

$$
\begin{aligned}
J_{1 n}+J_{2 n} & =\sum_{i=1}^{n} \prod_{j=1}^{i} E\left(1-X_{j}\right)^{2}+2 \sum \sum_{i<i^{*}} \prod_{j=1}^{i}\left(1-X_{j}\right)^{2} \prod_{l=i+1}^{i^{*}} E\left(1-X_{j}\right)^{i^{*}-1} \\
& =\sum_{i=1}^{n-1}\left(1-2 \alpha+\alpha_{(2)}\right)^{i}+2 \sum \sum_{i<i^{*}}\left(1-2 \alpha+\alpha_{(2)}\right)^{i}(1-\alpha)^{i^{*}-1}
\end{aligned}
$$

Now, as $n \rightarrow \infty$

$$
J_{1 n}=\frac{\left(1-2 \alpha+\alpha_{(2)}\right)\left[1-\left(1-2 \alpha+\alpha_{(2)}\right)^{n}\right]}{2 \alpha-\alpha_{(2)}} \rightarrow \frac{1-2 \alpha+\alpha_{(2)}}{2 \alpha-\alpha_{(2)}},
$$

and, after some calculations, we get that

$$
\begin{aligned}
J_{2 n}=\frac{2(1-\alpha)}{\alpha} & \sum_{i=1}^{n-1}\left(1-2 \alpha+\alpha_{(2)}\right)^{i} \\
& -\frac{2(1-\alpha)}{\alpha} \sum_{i=1}^{n-1}\left(\frac{1-2 \alpha+\alpha_{(2)}}{1-\alpha}\right)^{i} \frac{2(1-\alpha)\left(1-2 \alpha+\alpha_{(2)}\right)}{\alpha\left(2 \alpha-\alpha_{(2)}\right)},
\end{aligned}
$$

as $n \rightarrow \infty$. Hence as $n \rightarrow \infty$

$$
\operatorname{Var}\left(P_{\bar{n}}\right) \rightarrow
$$




$$
\begin{aligned}
\frac{\alpha^{2}\left(1-2 \alpha+\alpha_{(2)}\right)+2 \alpha(1-\alpha)\left(1-2 \alpha+\alpha_{(2)}\right)-}{\alpha^{2}\left(2 \alpha-\alpha_{(2)}\right)}(1-\alpha)^{2}\left(2 \alpha-\alpha_{(2)}\right) \\
=\frac{\alpha_{(2)}-\alpha^{2}}{\alpha^{2}\left(2 \alpha-\alpha_{(2)}\right)} .
\end{aligned}
$$

Theorem 2 is now proved.

Corollary 3. If $\lim _{n \rightarrow \infty} \underline{\mu}_{n}=\alpha, \lim _{n \rightarrow \infty} \bar{\mu}^{n}=\beta$, and, if $\alpha$ and $\beta$ are both positive, then $\lim _{n \rightarrow \infty} E\left(P_{\bar{n}}\right) \in\left[\frac{1-\beta}{\beta}, \frac{1-\alpha}{\alpha}\right]$. Furthermore, if $\lim _{n \rightarrow \infty} \underline{\mu}^{(2)}=\alpha^{(2)}$ and $\lim _{n \rightarrow \infty} \bar{\mu}_{n}^{(2)}=\beta^{(2)}$, then

$$
\begin{aligned}
& E\left(P_{\bar{n}}\right)^{2} \in\left[\frac{1-2 \beta+\alpha^{(2)}}{2 \beta-\alpha^{(2)}}+\frac{2(1-\beta)\left(1-2 \beta+\alpha^{(2)}\right)}{\beta\left(2 \beta-\alpha^{(2)}\right)},\right. \\
&\left.\frac{1-2 \alpha+\beta^{(2)}}{2 \alpha-\beta^{(2)}}+\frac{2(1-\alpha)\left(1-2 \alpha+\beta^{(2)}\right)}{\alpha\left(2 \alpha-\beta^{(2)}\right)}\right] .
\end{aligned}
$$

Note that if $\underline{\mu}_{n} \rightarrow \bar{\mu}, \bar{\mu}_{n} \rightarrow \bar{\mu}, \underline{\mu}_{n}^{(2)} \rightarrow \underline{\mu}^{(2)}$ and $\bar{\mu}_{n}^{(2)} \rightarrow \bar{\mu}^{(2)}$ as $n \rightarrow \infty$ and provided that $1-2 \underline{\mu}_{n}+\bar{\mu}_{n}^{(2)}<1$ and $1-2 \bar{\mu}_{n}+\underline{\mu}_{n}^{(2)}<1$ for all $n$, then we easily see from Theorem 2 Part (ii) that

$$
B \leq \lim _{n \rightarrow \infty} E\left(P_{\bar{n}}\right)^{2} \leq A,
$$

where $A=\frac{1-2 \underline{\mu}+\bar{\mu}^{(2)}}{2 \underline{\mu}-\bar{\mu}^{(2)}}+\frac{2(1-\underline{\mu})}{\underline{\mu}}\left(1-2 \underline{\mu}+\bar{\mu}^{(2)}\right)\left(\frac{1}{2 \underline{\mu}-\bar{\mu}^{(2)}}\right)$. B is obtained from $A$ by replacing $\underline{\mu}$ with $\bar{\mu}$ and $\bar{\mu}^{(2)}$ by $\underline{\mu}^{(2)}$.

Thus combining (2.5) and $(\overline{2} .16)$ we get that

$$
B-a^{2} \leq \operatorname{Var}\left(P_{\bar{n}}\right) \leq A-b^{2}
$$

Hence, in view of Chebychev inequality $n^{-\epsilon}\left(P_{m}-E\left(P_{m}\right)\right.$ converges in probability to 0 as $n \rightarrow \infty$ for any $\epsilon>0$. Further, in view of the Borel-Cantelli lemma $n^{-\epsilon}\left(P_{\bar{n}}-E\left(P_{\bar{n}}\right)\right.$ converges to 0 with probability 1 for all $\epsilon>\frac{1}{2}$. A more refined result in this direction is given in the next section.

\section{Strong Convergence Results}

In this section, we address the question: when does $P_{\bar{n}}-E\left(P_{\bar{n}}\right)$ converge to 0 with probability 1 . We provide an answer in the case when $X_{i}$ 's are bounded above and below (away from zero). In particular, we prove the following result. 
Theorem 4. Let $\alpha_{i} \leq X_{i} \leq \beta_{i}$ for $i=1,2, \ldots, n$ with probability 1 . Set $\underline{\alpha}_{n}=\min _{1 \leq i \leq n} \alpha_{i}$ and $\bar{\beta}_{n}=\max _{1 \leq \leq n} \beta_{i}$. If for any $\epsilon>0, \sum_{n=1}^{\infty} \exp \left[\frac{-\epsilon \bar{\beta}_{n}}{\underline{\alpha}_{n}^{2}}\right]<\infty$, then $P_{\bar{n}}-E\left(P_{\bar{n}}\right) \rightarrow 0$ with probability 1 as $n \rightarrow \infty$.

Proof. Note that as above $P_{\bar{n}}=\sum_{i=1}^{n} \prod_{l=1}^{i}\left(1-X_{l}\right)$, thus

$$
\beta_{n}^{*}=\frac{1-\bar{\beta}_{n}}{\bar{\beta}_{n}}\left[1-\left(1-\bar{\beta}_{n}\right)^{n}\right] \leq P_{\bar{n}} \leq \frac{1-\underline{\alpha}_{n}}{\underline{\alpha}_{n}}\left[1-\left(1-\underline{\alpha}_{n}\right)^{n}\right]=\alpha_{n}^{*} .
$$

Set $\alpha^{*}=\alpha_{n}^{*}$ and $\beta^{*}=\beta_{n}^{*}$. Thus, for any $t>0$ and any $\xi>0$

$$
P\left[\left|P_{\bar{n}}-E P_{\bar{n}}\right|>t\right] \leq e^{-t \xi-\xi E P_{\bar{n}}} E e^{\xi P_{\bar{n}}} .
$$

But, since $\beta^{*} \leq P_{n} \leq \alpha^{*}$, then for any $\xi>0$,

$$
E^{\xi P_{n}} \leq \frac{\alpha^{-} E P_{\bar{n}}}{\alpha^{*}-\beta^{*}} e^{\xi \beta^{*}}+\frac{E P_{\bar{n}}-\beta^{*}}{\alpha^{*}-\beta^{*}} e^{\xi \alpha^{*}} .
$$

Set

$$
G(\xi)=-\xi E P_{\bar{n}}+\ln \left[\frac{\alpha^{*}-E P_{\bar{n}}}{\alpha^{*}-\beta^{*}} e^{\xi \beta^{*}}+\frac{E P_{\bar{n}}-\beta^{*}}{\alpha^{*}-\beta^{*}} e^{\xi \alpha^{*}}\right] .
$$

Further let

$$
\begin{array}{r}
T_{n}=\frac{E P_{\bar{n}}-\beta^{*}}{\alpha^{*}-\beta^{*}} \text { and } \xi_{n}=\xi\left(\alpha^{*}-\beta^{*}\right), \text { for all } n \geq 1, \text { then } \\
G\left(\xi_{n}\right)=-\xi_{n} T_{n}+\ln \left[1-T_{n}+T_{n} e^{\xi_{n}}\right] .
\end{array}
$$

Using Taylor expansion, we get that

$G\left(\xi_{n}\right)=G(0)+G^{\prime}(0) \xi_{n}+G^{n}(\theta) \xi_{n}^{2}$, where $0 \leq \theta \leq \xi_{n}$.

But,

$$
G^{\prime}\left(\xi_{n}\right)=-T_{n}+\frac{T_{n}}{\left(1-T_{n}\right) e^{-\xi_{n}}+T_{n}}
$$

and

$$
G^{\prime \prime}\left(\xi_{n}\right)=\frac{T_{n}\left(1-T_{n}\right) e^{-\xi_{n}}}{\left[\left(1-T_{n}\right) e^{-\xi_{n}}+T_{n}\right]^{2}}
$$

Thus

$$
G^{\prime \prime}\left(\xi_{n}\right)=\left[\frac{T_{n}}{\left(1-T_{n}\right) e^{-\xi_{n}}+T_{n}}\right]\left[1-\frac{T_{n}}{\left(1-T_{n}\right) e^{-\xi_{n}}+T_{n}}\right] .
$$

Hence $G^{\prime \prime}\left(\xi_{n}\right) \leq \frac{1}{4}$ for any $\xi_{n}$ and therefore, 


$$
G\left(\xi_{n}\right) \leq G(0)+G^{\prime}(0) \xi_{n}+\frac{\xi_{n}^{2}}{8}=\frac{\xi_{n}^{2}}{8}
$$

Hence,

$$
E e^{\xi\left(P_{\bar{n}}-E P_{\bar{n}}\right)} \leq e^{\xi^{2}\left(\alpha^{*^{2}}-\beta^{*^{2}}\right) / 8}
$$

Therefore,

$$
P\left[\left|P_{\bar{n} \mid}-E P_{\bar{n}}\right|>t\right] \leq e^{-t \xi+\xi^{2}\left(\alpha^{*^{2}}-\beta^{*^{2}}\right) / 8}
$$

Set

$$
f(\xi)=-t \xi+\xi^{2}\left(\alpha^{*^{2}}-\beta^{*^{2}}\right) / 8
$$

then $f^{\prime}(\xi)=0$ gives $\xi=\frac{4 t}{\alpha^{*^{2}}-\beta^{*^{2}}}$, as minimum value and hence we get

$$
P\left[\left|P_{\bar{n}}-E P_{\bar{n}}\right|>t\right] \leq e^{-2 t^{2} /\left(\alpha^{*^{2}}-\beta^{*^{2}}\right)} .
$$

But,

$$
\begin{aligned}
\alpha^{*}-\beta^{*} & =\sum_{i=1}^{n}\left[\left(1-\underline{\alpha}_{n}\right)^{i}-\left(1-\bar{\beta}_{n}\right)^{i}\right] \\
& =\sum_{i=1}^{n}\left(\bar{\beta}_{n}-\underline{\alpha}_{n}\right)\left[\left(1-\underline{\mu}_{n}\right)^{i-1}+\left(1-\underline{\alpha}_{n}\right)^{i-2}\left(1-\bar{\beta}_{n}\right)+\left(1-\bar{\beta}_{n}\right)^{i-1}\right] \\
& \leq\left(\bar{\beta}_{n}-\underline{\alpha}_{n}\right) \sum_{i=1}^{n} i\left(1-\underline{\alpha}_{n}\right)^{i-1} \\
& =\frac{\bar{\beta}_{n}-\underline{\alpha}_{n}}{\underline{\alpha}_{n}^{2}} \\
& \leq \frac{\bar{\beta}_{n}}{\underline{\alpha}_{n}^{2}} .
\end{aligned}
$$

Finally,

$$
\alpha^{*^{2}}-\beta^{*^{2}}=\left(\alpha^{*}-\beta^{*}\right)\left(\alpha^{*}+\beta^{*}\right) \leq \frac{2\left(\alpha^{*}-\beta^{*}\right)}{\underline{\alpha}_{n}} \leq \frac{2 \bar{\beta}_{n}}{\underline{\alpha}_{n}^{3}} .
$$

The theorem is proved in view of the Borel-Cantelli lemma.

For example, we can apply Theorem 4 when we take for all $i=1,2, \ldots, n$, $\frac{C_{1}}{n} \leq X_{i} \leq \frac{C_{2}}{n}$ where $C_{1}<C_{2}$. 


\section{References}

[1] N. I. Bowers, H. U. Gerber, J. C. Hickman, D. A. Jones, D. A. and C. J. Nebitt. em Actuarial Mathematics, second edition, Society of Actuaries, schaumburg, IL. U.S.A. (1997)

[2] P. P. Boyle, Rates of return as random variables. Journal of Risk and Insurance 53 (4), (1976), 693-713.

[3] K. Burnecki, A. Marciniuk, and A. Weron, A. Annuities under random rates of interest-Revisited. Insurance: Mathematics and Economics 32, (2003) 457-460.

[4] D. Dufresne, Stochastic life annuities. N. Am. Actuar. Journal 11 (1), (2007). 136-157.

[5] S. G. Kellieson, The theory of interest. McGraw-Hill, New York, NY. U.S.A. (1997).

[6] A. Kling, F. Ruez, and J. Rub, The impact of stochastic volatility on pricing, hedging, and hedge efficiency of withdrawal benefit guarantees in variable annuities. Astin Bull 41, 2, (2011) 511-545.

[7] H. Panjer, and D. Bellhouse. Stochastic modeling of interest rates with application to life contingencies. Journal of Risk and Insurance 47, (1980). 91-119.

[8] H. Panjer, and D. Bellhouse. Stochastic modeling of interest rates with application to life contingencies - Part II. Journal of Risk and Insuarance 48, (1981) 728-637.

[9] S. D. Promislow. A new approach to the theory of interest. Transactions of Society of Actuaries. 32, (19810) 53-92. 